\title{
Red GPS Topo-Iberia: Resultados obtenidos en el Centro de Análisis de la UJA
}

\author{
M. Clara de LACY PÉREZ De LOS COBOS ${ }^{1,2}$, JuAN ANTONIO ARMENTEROS ${ }^{1,2}$, \\ ANTONIO. M. RUIZ ARMENTEROS ${ }^{1,2}$, ANTONIO J. GIL CRUZ ${ }^{1,2}$, \\ FEDERICA RIGUZZI $^{3}$, ROBERTO DEVOTI ${ }^{3}$ \\ Y GRUPO GPS DE TOPO-IBERIA \\ ${ }^{1}$ Dpto. Ingeniería Cartográfica, Geodésica y Fotogrametría. Universidad de Jaén (Spain) \\ ${ }^{2}$ Centro de Estudios Avanzados de Ciencias de la Tierra (Universidad de Jaén) \\ mclacy@ujaen.es \\ ${ }^{3}$ Istituto Nazionale di Geofisica e Vulcanologia, sez. CNT. Roma, Italy \\ federica.riguzzi@ingv.it
}

Recibido: 4/09/2014

Aceptado: 6/10/2014

\begin{abstract}
Resumen
El proyecto "Geociencias en Iberia: Estudios integrados de topografía y evolución 4D: TopoIberia" (Ref.CSD2006-00041) fue financiado por el Ministerio de Economía y Competitividad. Su objetivo era entender las interacciones en la Península Ibérica entre procesos atmosféricos profundos y de superficie, a través de un estudio multidisciplinar que unía ciencias como la Geología, Geofísica y Geodesia. Dentro de este trabajo se estableció una red de 26 estaciones GPS permanentes, de las cuales 22 cubren la parte española de la Península Ibérica y las otras 4 están localizadas en Marruecos. Esta red que empezó a funcionar en 2008, complementa otras redes de estaciones GPS permanentes que pertenecen a instituciones internacionales como EPN (EUREF Permanent Network), IGS (International GNSS Service) y a instituciones regionales. La finalidad de todas ellas es el control de deformaciones. En este trabajo se aplica al caso particular del movimiento relativo de las placas tectónicas de Eurasia y Nubia. Del análisis de los datos se ocupan tres centros: el Real Instituto y Observatorio de la Armada (ROA), la Universidad de Barcelona (UB) y la Universidad de Jaén (UJA). En este artículo se presentan las series temporales y campo de velocidades calculados en la UJA con datos desde el 1 de marzo de 2008 hasta el 31 de diciembre de 2013. Las velocidades estimadas ponen de manifiesto que el Sureste de la Península Ibérica es una zona de frontera entre dos placas, que acomoda los movimientos relativos entre las placas de Eurasia y Nubia
\end{abstract}

Palabras clave: control de deformaciones, red GPS permanente, campo de velocidades.

\section{Topo-Iberia GPS network: results obtained at UJA Analysis Centre}

\begin{abstract}
The project "Geosciences in Iberia: Integrated studies of topography and 4-D evolution Topo-Iberia" (Ref.CSD2006-00041) is supported by the Spanish Ministry for the Economy and Competitiveness. Its objective is to understand the interactions in the Iberian Peninsula (SW Europe) between deep, shallow and atmospheric processes, through a multidisciplinary approach linking Geology, geophysics and Geodesy. As part of this project a network of 26 CGPS (continuous GPS) stations, covering the Spanish part of the Iberian Peninsula (22 stations) and Morocco (4 stations) was established in 2008 to complement other GPS networks already deployed by different Institutions, EPN (EUREF Permanent Network), IGS (InternationalGNSS Service) and regional governmental agencies to monitor crustal
\end{abstract}


deformation due to Nubia and Eurasian tectonic plates. Data analysis is performed at three different analysis centres: Real Instituto y Observatorio de la Armada (ROA), University of Barcelona (UB) and University of Jaen (UJA). The coordinate time series and the velocity field from 2008-2013 computed at UJA analysis centre are presented in this paper. The estimated velocities show the southeast of the Iberian Peninsula is a boundary area between two plates accommodating the relative movements between the Eurasian and Nubia plates.

Key words: Deformation monitoring, permanent GPS network, velocity field.

Sumario: Introducción. 1. Red GPS permanente Topo-Iberia. 2. Procesado de datos en la UJA. 3. Campo de velocidades GPS. 4. Conclusiones. Agradecimientos. Referencias Bibliográficas.

\section{Referencia normalizada}

Lacy M.C de, Armenteros J. A., Ruiz A. M., Gil A. J., Riguzzi F., Devoti R. (2014). Red GPS TopoIberia: Resultados obtenidos en el Centro de Análisis de la UJA. Física de la Tierra, Vol. 26, 25-33.

\section{Introducción}

La península Ibérica y sus márgenes, con un relieve que evidencia la intensidad y gran dispersión de las deformaciones tectónicas recientes, constituye un laboratorio natural idóneo para desarrollar investigaciones sobre su topografía y evolución 4-D. El objetivo del proyecto "Geociencias en Iberia: Estudios integrados de topografía y evolución 4D: Topo-Iberia" es comprender la interacción entre procesos profundos, superficiales y atmosféricos, integrando las investigaciones en Geología, Geofísica y Geodesia.

El empleo de técnicas GPS para el control de deformaciones del terreno, permite la monitorización de áreas tectónicamente activas y la detección de movimientos relativos de pequeña magnitud (por debajo del centímetro), como sucede en las zonas de la península Ibérica (Avallone et al 2010; Devoti et al 2011; Fadil et al 2006; Marín-Lechado et al 2010; McClusky et al 2003). Así, con el fin de monitorizar las deformaciones milimétricas de la corteza debidas a los movimientos de las placas tectónicas de Eurasia y Nubia, se ha instalado una red de 26 estaciones GPS permanentes estratégicamente situadas: 22 estaciones en la parte española de la Península Ibérica y 4 en Marruecos. Un objetivo fundamental del proyecto es la determinación del vector de deformaciones actual de la corteza. Dentro de otros objetivos del proyecto se incluye también la identificación de áreas y fallas sísmicas específicas que presenten mayores velocidades de deformación, lo que supondría un mayor riesgo sísmico en dichas zonas.

\section{Red GPS permanente Topo-Iberia}

Una serie de nuevas estaciones GPS permanentes se han instalado como complemento a otras redes GPS pertenecientes a diferentes instituciones (EUREF, IGS y Agencias Regionales de Gobierno). Después de un período inicial de diseño, adquisición de nuevos equipos, monumentación y puesta en marcha de los mismos, en la actualidad la red GPS de Topo-Iberia consta de veintiséis estaciones operativas (Fig. 1). Estos sistemas autónomos se han diseñado incluyendo un receptor GPS y una 
antena GNSS Choke-Ring, un panel solar, dos baterías y un módem GPRS (Fig. 2). Como norma general, los equipos se han instalado sobre el terreno, situando las antenas en pilares de hormigón sobre afloramientos rocosos. Sólo en casos donde las condiciones ambientales lo aconsejaban, se han empleado radomos protectores de antena, como es el caso de la estación NEVA situada en el Observatorio de Sierra Nevada (Granada).

El funcionamiento de las primeras estaciones de la red se inició el 1 de marzo de 2008, estando todas las estaciones operativas en diciembre del mismo año. 22 estaciones se encuentran en diferentes regiones de España y las otras 4 están ubicadas en lugares estratégicamente seleccionados en el norte de Marruecos para completar la visión general de la cinemática actual en la Península Ibérica y el sur de las áreas vecinas, incluyendo la cadena del Atlas.

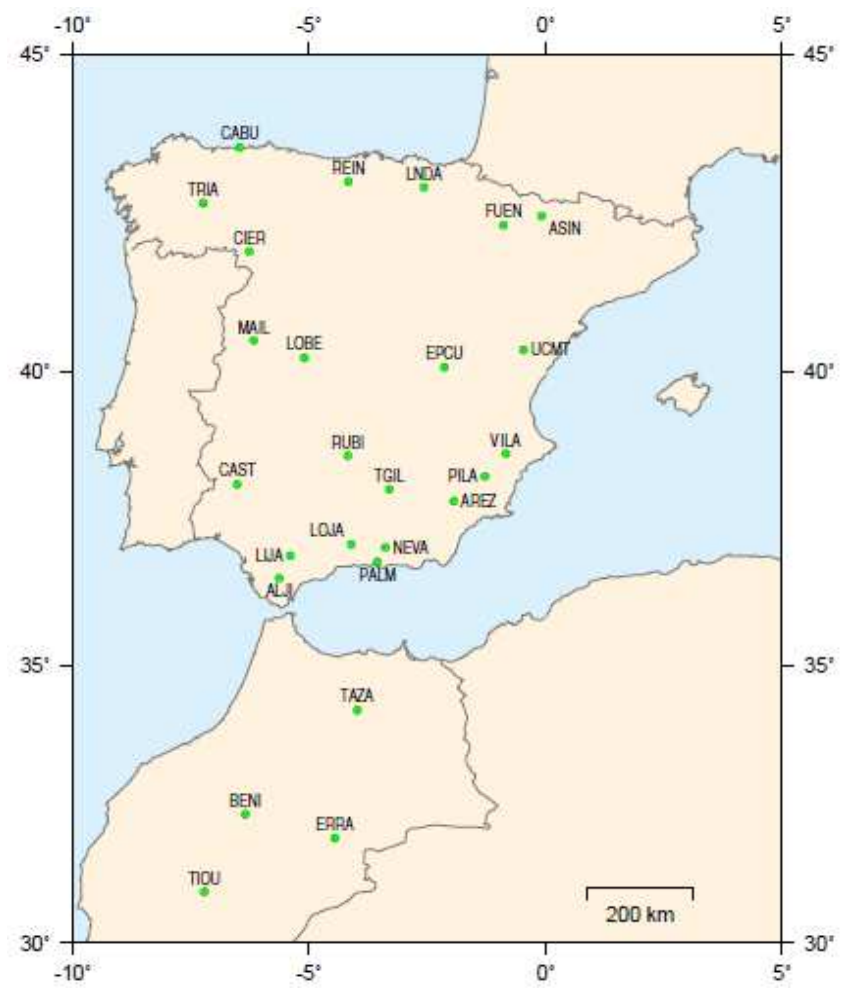

Fig. 1. Mapa de estaciones GPS de Topo-Iberia. 


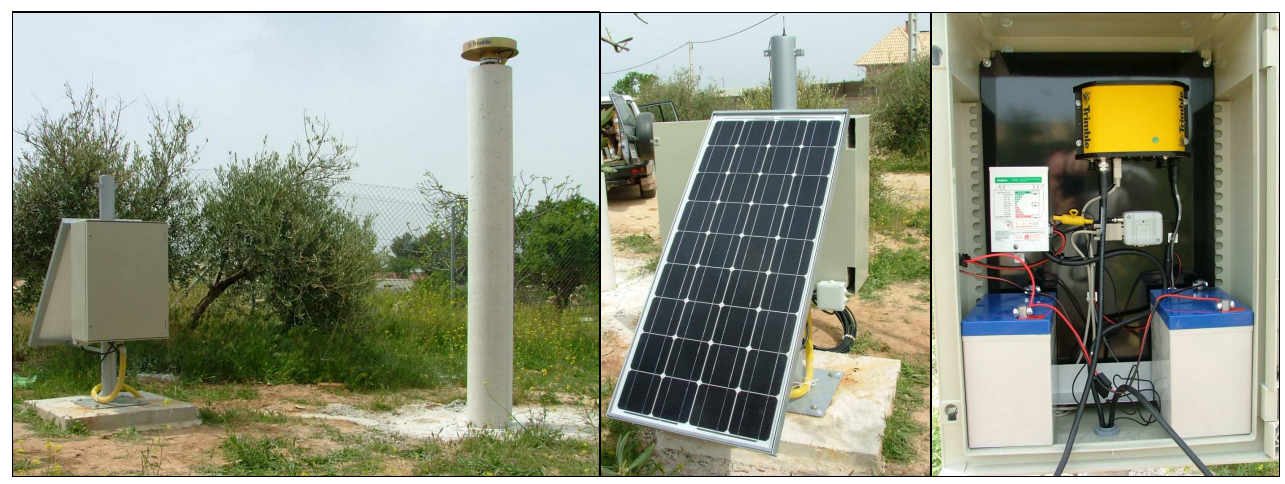

Fig. 2. Estación TGIL, vista general, panel solar y receptor GPS.

\section{Procesado de datos en la UJA}

En este trabajo se han procesado de manera independiente con el mismo procedimiento dos clusters de datos GPS: uno de ellos con las estaciones de Topo-Iberia desde el 1 de marzo de 2008 hasta el 31 de diciembre de 2013 y otro con datos de estaciones permanentes GPS europeas pertenecientes a EUREF e IGS desde 1998 hasta 2013. Este cluster ha sido generado por el Istituto Nazionale di Geofisica e Vulcanología (INGV) (Devoti et al 2008). Esta decisión se tomó en el año 2009 para conseguir un conjunto de estaciones fiduciales más estable. El análisis de los datos en la UJA se realiza con el programa científico Bernese 5.0 (Dach et al., 2007), usando ambos clusters las mismas opciones. En particular, utiliza las dobles diferencias de fase como observables; las órbitas precisas del IGS y los parámetros de orientación de la Tierra se mantienen fijos, aplicándose las correcciones absolutas de centro de fase -dependientes de la elevación del satélite- proporcionadas por el IGS (Dow et al, 2005). Se estima una solución diaria en un marco de referencia débilmente constreñido, cercano a la condición de deficiencia de rango. Esta solución poco constreñida es calculada en un marco de referencia intrínseco, definido por las propias observaciones, que se diferencia de un día a otro por las traslaciones de red rígidas, manteniendo las distancias entre estaciones siempre bien definidas. Los constreñimientos para la realización del marco de referencia escogido son impuestos sólo a posteriori. A continuación, las soluciones diarias débilmente constreñidas procedentes de los distintos clusters se fusionan en soluciones globales mínimamente constreñidas de toda la red aplicando la teoría clásica de mínimos cuadrados (Bianco et al., 2003). Por último, estas soluciones mínimamente constreñidas se han llevado al marco de referencia IGb08. En particular se ha usado la realización EPN_A_IGb08 con las siguientes estaciones fiduciales: AJAC, BOR1, BUCU, CAGL, EBRE, GRAS, GRAZ, LAMP, MATE, NOT1, SFER, SJDV y ZIMM. 
El campo de velocidades se estima mediante el uso de un software especialmente diseñado para ello (NEVE) (Devoti et al 2008), desarrollado por el Istituto Nazionale di Geofisica e Vulcanologia di Roma (Italia), que trabaja con un modelo estocástico completo. Dicho campo se calcula a partir de las series temporales en el marco de referencia considerado. Simultáneamente se calculan las velocidades junto con las componentes anuales y los desplazamientos ocurridos en épocas de cambios instrumentales. Los errores asociados a las velocidades se derivan de la propagación directa de las matrices de covarianza diarias. Las series temporales se han calculado con los datos disponibles de las estaciones GPS desde su puesta en funcionamiento el 1 de marzo de 2008 hasta el 31 de Diciembre de 2013 (Fig. 3).
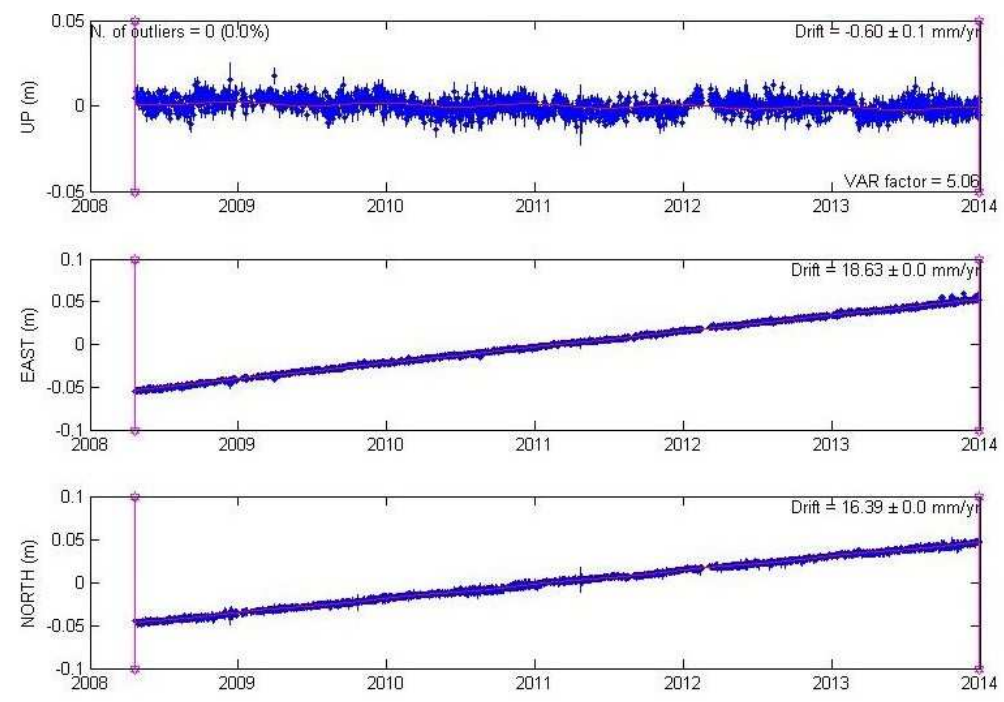

Fig. 3. Componentes altimétrica y planimétricas de la serie temporal de la estación de TGIL en IGb08.

\section{Campo de velocidades GPS}

La determinación del campo de velocidades permite conocer las características de la deformación actual de la corteza terrestre en la Península Ibérica. Las velocidades absolutas respecto del marco de referencia considerado pueden verse en la Figura 4. 


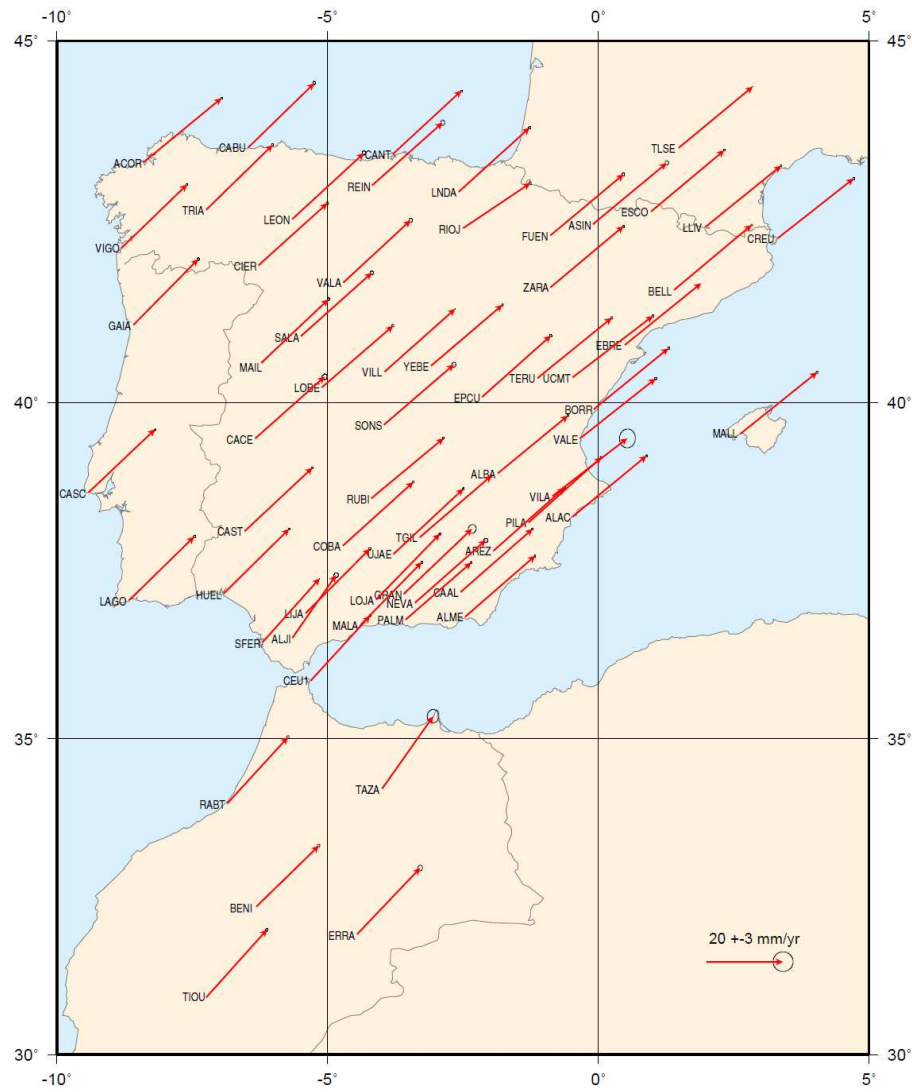

Fig. 4. Campo de Velocidades del cluster español en IGb08

Las velocidades residuales respecto a la placa de Eurasia han sido derivadas a partir de las velocidades absolutas utilizando los párametros publicados en (Altamimi et al 2011). Un ejemplo de las velocidades obtenidas para algunas de las estaciones procesadas puede verse en la Tabla 1. La Figura 5 muestra las velocidades residuales de la red Topo-Iberia completa.

Tabla. 1. Un ejemplo de las velocidades obtenidas respecto a la placa Euroasiática en $\mathrm{mm} / \mathrm{año}$. Las estaciones marcadas con ${ }^{(*)}$ pertenecen a la red Topo-Iberia

\begin{tabular}{|c|c|c|}
\hline Estación & Vel. Este & Vel. Norte \\
\hline ACOR & $3.77 \pm 0.45$ & $0.59 \pm 0.45$ \\
\hline AREZ $^{(*)}$ & $-1.26 \pm 0.46$ & $0.77 \pm 0.45$ \\
\hline BOR1 & $0.34 \pm 0.46$ & $-0.37 \pm 0.44$ \\
\hline $\mathrm{EPCU}^{(*)}$ & $-0.70 \pm 0.46$ & $1.19 \pm 0.46$ \\
\hline $\mathrm{ERRA}^{(*)}$ & $-4.20 \pm 0.47$ & $2.67 \pm 0.45$ \\
\hline
\end{tabular}




\begin{tabular}{|c|c|c|}
\hline GLSV & $-0.35 \pm 0.47$ & $-0.40 \pm 0.44$ \\
\hline GRAS & $0.69 \pm 0.46$ & $0.33 \pm 0.45$ \\
\hline GRAZ & $0.78 \pm 0.46$ & $0.86 \pm 0.45$ \\
\hline LIJA $^{(*)}$ & $-2.39 \pm 0.46$ & $0.97 \pm 0.45$ \\
\hline LLIV & $0.57 \pm 0.46$ & $0.54 \pm 0.45$ \\
\hline MALA & $-2.14 \pm 0.46$ & $0.05 \pm 0.45$ \\
\hline MARS & $0.29 \pm 0.46$ & $0.59 \pm 0.45$ \\
\hline PILA $^{(*)}$ & $-0.85 \pm 0.46$ & $1.63 \pm 0.45$ \\
\hline SFER & $-3.85 \pm 0.46$ & $1.03 \pm 0.50$ \\
\hline SJDV & $0.40 \pm 0.46$ & $0.28 \pm 0.44$ \\
\hline TAZA $^{(*)}$ & $-4.13 \pm 0.48$ & $3.15 \pm 0.48$ \\
\hline TGIL $^{(*)}$ & $-0.91 \pm 0.46$ & $0.32 \pm 0.45$ \\
\hline $\mathrm{TIOU}^{(*)}$ & $-4.4 \pm 0.47$ & $2.71 \pm 0.46$ \\
\hline ZIMM & $0.55 \pm 0.46$ & $0.73 \pm 0.44$ \\
\hline
\end{tabular}

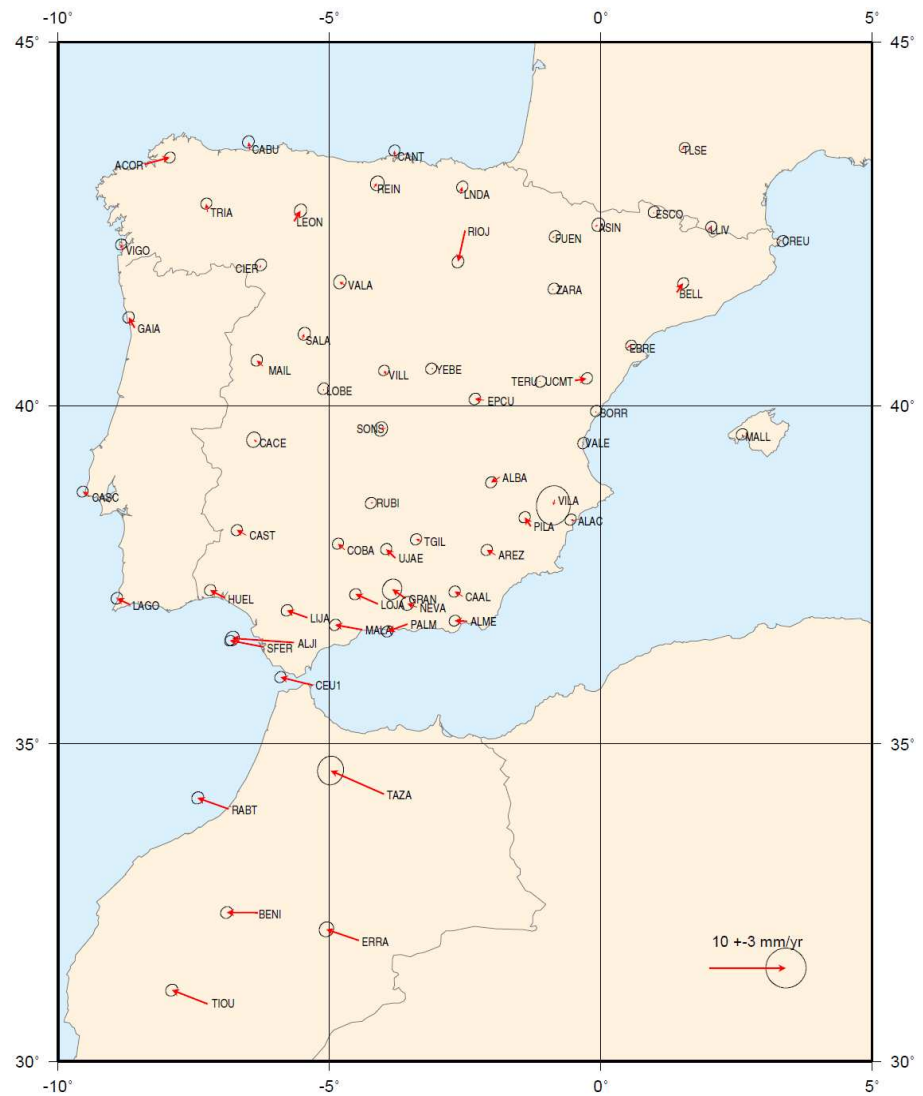

Fig. 5. Velocidades del cluster español respecto a la placa Euroasiática 


\section{Conclusiones}

La mayoría de los puntos muestran velocidades muy pequeñas respecto a la placa de Eurasia. Una excepción es ACOR. La razón no está muy clara aunque podría estar ligada a problemas del receptor de esta estación. El Sistema Bético es la zona más activa tectónicamente de la Península Ibérica, hecho que se pone de manifiesto en los resultados obtenidos. Algunos de los correspondientes valores pueden verse en la Tabla 1, como por ejemplo LIJA y SFER. Tal y como era de esperar, a partir de las velocidades estimadas se pone de manifiesto que el Sureste de la Península Ibérica es una zona de frontera entre dos placas, que acomoda los movimientos relativos entre las placas de Eurasia y Nubia. La zona de mayor movimiento se localiza en el Norte de Marruecos, siendo ejemplo de ello las velocidades de las estaciones de ERRA y TIOU. Por otro lado las estaciones situadas en la parte estable de la placa euroasiática muestran velocidades con valores cercanos a cero, tal y como puede verse en las estaciones de BOR1, GLSV, GRAS, GRAZ, MARS, SJDV y ZIMM. Por último, es importante resaltar la homogeneidad de los errores cuadráticos medios de las velocidades estimadas.

\section{Agradecimientos}

Esta investigación ha sido financiada por el Programa Consolider-Ingenio 2010, dentro del proyecto Topo-Iberia CSD2006-0041 del Ministerio de Economía y Competitividad de España. Algunas figuras fueron hechas usando Generic Mapping Tools (Wessel and Smith, 1998).

\section{Referencias Bibliográficas}

ALTAMIMI Z, COLLILIEUX X, MÉTIVIER L (2011). ITRF2008: an improved solution of the international terrestrial reference frame. J Geod 85:457-473

AVALLONE A, SELVAGGI G, D'ANASTASIO E et al (2010). The RING network: improvement of a GPS velocity field in the central Mediterranean. Ann Geophys 53:39-54.

BIANCO, G., DEVOTI, R., LUCERI, V. (2003). Combination of loosely constrained solutions. IERS Tech. Note 30, 107-109.

DACH, R., U. HUGENTOBLER, P. FRIDEZ, AND M. MEINDL, Eds. (2007). Bernese GPS Software, Version 5.0, User Manual, Astronomical Institute, University of Bern. Bern. Switzerland.

DEVOTI, R., RIGUZZI, F., CUFFARO, M., DOGLIONI, C. (2008). New GPS constraints on the kinematics of the Apennine subduction. Earth and Planetary Science Letters, 273 (1-2), 163-174.

DEVOTI R, ESPOSITO A, PIETRANTONIO G et al. (2011). Evidence of large scale deformation patterns from GPS data in the Italian subduction boundary. Earth Planet Sci Lett 311:230-241. 
DOW, J.M.; NEILAN, R.E.; GENDT, G. (2005). The International GPS Service (IGS): Celebrating the 10th Anniversary and Looking to the Next Decade, $A d v$. Space Res., 36, 320-326.

FADIL A, VERNANT P, MCCLUSKY S et al. (2006). Active tectonics of the western Mediterranean: geodetic evidence for rollback of a delaminated subcontinental lithospheric slab beneath the Rif Mountains, Morocco. Geology 34:529-532

MCCLUSKY S, REILINGER R, MAHMOUD S et al. (2003). GPS constraints on Africa (Nubia) and Arabia plate motions. Geophys J Int 155:126-138.

MARÍN-LECHADO C, GALINDO-ZALDÍVAR J, GIL AJ et al. (2010). Levelling profiles and a GPS network to monitor the active folding and faulting deformation in the Campo de Dalias (Betic Cordillera, Southeastern Spain). Sensors 10:3504-3518.

WESSEL, P. AND W. H. F. SMITH (1998). New improved version of the Generic Mapping Tools released, EOS Trans. AGU, 79, 579. 